\title{
e-Phaïstos
}

e-Phaïstos

Revue d'histoire des techniques / Journal of the history

of technology

IV-1 | 2015

Les arts de guerre et de grâce (XIVe-XVIIIe siècles)

\section{François Ruegg, La maison paysanne, histoire et mythe}

\section{Ivan Lafarge}

\section{OpenEdition}

\section{Journals}

Édition électronique

URL : http://journals.openedition.org/ephaistos/675

DOI : 10.4000/ephaistos.675

ISSN : 2552-0741

\section{Éditeur}

IHMC - Institut d'histoire moderne et contemporaine (UMR 8066)

\section{Édition imprimée}

Date de publication : 1 avril 2015

Pagination : 98-104

ISSN : 2262-7340

\section{Référence électronique}

Ivan Lafarge, «François Ruegg, La maison paysanne, histoire et mythe », e-Phaïstos [En ligne], IV-1 ]

2015, mis en ligne le 24 novembre 2016, consulté le 24 septembre 2020. URL : http://

journals.openedition.org/ephaistos/675 ; DOI : https://doi.org/10.4000/ephaistos.675 


\title{
François Ruegg, La maison paysanne : histoire d'un mythe
}

(collection Archigraphy, éditions Infolio, Gollion, 2011, 304 pages)

\author{
Ivan Lafarge \\ Docteur en histoire \\ Équipe d'Histoire des Techniques / \\ IHMC \\ CNRS - Université Paris 1 Panthéon- \\ Sorbonne
}

\begin{abstract}
Ce livre présente à travers six chapitres la construction de la notion de « maison paysanne » depuis le XVIIIe siècle ${ }^{1}$. Si la «maison des autres » a toujours intéressé, elle reste un sujet marginal de l'anthropologie sociale : la "maison traditionnelle" est souvent considérée comme le reflet de la culture locale, mais historiens et architectes lui préfèrent les monuments plus importants et pour l'ethnologue, elle reste trop souvent encore un objet de la culture matérielle à muséographier (chapitre 1). Il ne s'agit pas dans cet ouvrage d'ajouter au savoir encyclopédique relatif à l'architecture vernaculaire, mais de voir quand et comment sont nés les concepts d'architecture paysanne et populaire et à quoi ils correspondent en les remettant dans leur contexte idéologique, selon une perspective d'anthropologie sociale et culturelle. François Ruegg propose pour ce faire de développer une vision ethnohistorique. Il met en évidence à quel point "la maison paysanne" comme emblème des cultures nationales est une construction intellectuelle, même idéologique contemporaine. A travers les représentations, l'imaginaire qu'inspirent la maison rurale, ou les
\end{abstract}

rôles qu'on lui attribue, il cherche à reconstituer la cosmologie, la sociologie et l'histoire des idées liées aux diverses conceptions de l'habitat ou de l'habiter.

La formule "architecture sans architecte" (p.3339), rejoint les clichés à propos des sauvages, peuples «sans histoire, sans écriture et sans religion qu'on dit également sans art », parmi lesquels l'architecture au sens d'art développé est considérée comme absente. L'ethnographie montre qu'il n'y a pas de société sans racines, dont le fonctionnement ne repose pas au moins sur des mythes dont l'historicité a pour fonction d'expliquer l'origine ${ }^{2}$. Au delà de la réduction visant à considérer l'habiter comme un regroupement de faits techniques, l'ouvrage propose de remettre en question le mythe de la "maison paysanne" qui n'est pas l'expression d'une vérité intemporelle, et s'inscrit au contraire dans un contexte historique qu'on oublie trop souvent de prendre en compte. Les circonstances de la naissance de la "maison paysanne" sont expliquées au travers de la tradition germanique et de l'expansion de ses modèles en Europe orientale, dans une région marquée par l'obsession nationale de régimes 
sans cesse bouleversés par les révisions de frontières (chapitre 5).

La maison n'est pas considérée comme digne d'intérêt avant la fin du XIX ${ }^{\mathrm{e}}$ siècle. En France, Eugène Viollet-le-Duc en fait une analyse fortement marquée par sa propre idéologie et place la maison moderne occidentale au sommet d'une perspective évolutionniste 3 . La maison rurale ne devient objet d'étude qu'avec l'avènement de l'ethnographie rurale et du folklore ; alors que l'habitation fait depuis longtemps l'objet d'études de la part des architectes et de travaux d'encyclopédistes ${ }^{4}$. Dès la fin du XIX ${ }^{\mathrm{e}}$ siècle, chaque nation se dote d'un atlas ethnographique comprenant les relevés des principaux types de maisons rurales. Il y a dans l'ensemble une "nationalisation" des discours sur l'architecture populaire comme s'il n'existait aucune parenté entre la maison rurale du pays de Gex, française et la maison rurale genevoise séparées par une frontière politique récente. Il y a moins de différence entre ces deux là qu'entre la maison rurale du pays de Gex et celle d'Aquitaine ou la longère normande5. La Suisse est un exemple de cette construction intellectuelle et c'est d'ailleurs à partir de cette fiction paysanne qu'est né le chalet Suisse, " cet être qui ne ressemble à aucune maison rurale mais en est une sorte d'idée platonicienne dont la fortune ne cessera jamais... » (p.20-21). Il faut attendre 1969 pour que l'architecte Amos Rapoport ${ }^{6}$ ait une approche anthropologique de la maison.

L'architecture vernaculaire renverrait à un modèle ethnique ou protonational, l'idée que le changement n'intervient que dans les sociétés à histoire est si répandue que le modèle traditionnel est ainsi donné pour sans âge ou éternel. Malgré sa vacuité, cette idée est renforcée par la tendance à vouloir se rattacher à un modèle ancestral inchangé depuis la nuit des temps. L'architecture vernaculaire s'en trouve comme une catégorie intermédiaire entre architecture "naturelle" et architecture populaire qui ethnicise et nationalise la maison rurale pour en exploiter le potentiel imaginaire et sentimental au profit de la patrie (chapitre 5). Les discours folklo- riste et patrimonial sont ainsi utilisés pour construire une illusoire unité nationale. La représentation, stéréotype de la "maison paysanne" renvoie à une idée fonctionnelle où la notion "d'habitat primitif", reste problématique. L'auteur cherche donc à remettre en perspective les concepts idéologiques liés à l'architecture vernaculaire dans une approche pluridisciplinaire : anthropologique, archéologique, historique, "technologique". Il rend hommage au rôle d'André Leroi-Gourhan dans l'étude de l'environnement technique de l'habiter et lui reconnaît le mérite de l'approche ethnologique transcendant l'approche technique7. Selon Ruegg, les archéologues seraient à la recherche de "types primitifs » qui auraient servi de modèles ultérieurement ; en fait il s'agit d'une vision réductrice de l'approche archéologique dont la référence est malheureusement trop restreinte à la recherche mythifiée des « origines de la maison » dans la préhistoire (p.93101). Selon nous, cette approche devrait s'appuyer sur une analyse archéologique des maisons conservées à tous points de vue (modes de construction ; évolution et différenciation typologique ; distributions ; modes de chauffage, stockage, production ; rapport avec les pratiques culturales ; adaptations et incohérences relatives au terrain) plutôt que sur la recherche de "types primitifs". L'inconvénient de l'approche archéologique tient surtout à la difficulté de percevoir la complexité du fonctionnement social et son rapport avec les objets matériels.

Le chapitre 3 , en s'appuyant sur les caractères constructifs et techniques met en avant que la maison rurale est un objet social et politique, l'expression de phénomènes historiques et d'influences apportées par l'évolution d'une région. En tant qu'artefact relevant du croisement des cultures populaires, des mouvements politiques et des traditions locales elle est l'effet de volontés et de décisions plus ou moins conscientes fondées sur des représentations collectives et l'imaginaire populaire. Il ne s'agit pas de "génie paysan" mais d'un ensemble de "savoir-faire" étroitement mêlés, pas toujours sensibles dans la forme générale extérieure 
des constructions, mais qu'on décèle par l'examen structurel du bâti, ainsi les caves, les escaliers, les charpentes renvoient dans leur conception et leurs mises en oeuvre à des savoirs parfaitement compatibles avec le monde rural qui n'en sont pas moins des métiers en soi $^{8}$. Cela n'enlève rien à certaines spécificités de l'habitat rural au regard du milieu urbain : fonctions particulières du bâti, gestion différenciée de l'espace, forme et organisation des bâtiments.

L'Europe centrale-orientale a fait l'objet des conquêtes ottomane puis autrichienne. L'Aufklaerung ${ }^{9}$ donnait à la population une forte importance politique et stratégique. La construction traditionnelle de ces régions dans leur contexte d'isolement était dite à la turque au XVIIIe siècle "comme si cet adjectif pouvait décrire de manière satisfaisante une organisation particulière de l'espace et une technique de construction!"10. En 1906, la première publication consacrée à la "maison paysanne » (Bauernhaus) ${ }^{11}$ de l'empire austro-hongrois par une association d'architectes et d'ingénieurs est une commande politique de haute teneur qui bénéficie de la participation financière directe de l'Empereur François-Joseph : dès la fin du XIXe siècle, s'est ainsi développé "l'esprit national" auquel le style architectural est censé faire référence (chapitre 5).

Vaclav Frolec, l'un des spécialistes tchèques de l'architecture populaire les plus connus d'aprèsguerre lance l'hypothèse d'une progressive diversification (nationalisation) de la culture et de l'architecture populaires, alors qu'au Moyen Age on aurait eu affaire à une architecture bien plus homogène et élémentaire en Europe, qu'il s'agisse des techniques de construction ou du plan ${ }^{12}$; fantasme facilité par l'impression de lissage que provoque l'éloignement culturel.

En Scandinavie, les premiers exemples de considération de la maison rurale donnent lieu à des monographies dans lesquelles à un habitat germanique, on oppose un habitat slave dans l'idée d'une "maison nationale" reposant sur la théorie de l'autochtonie des peuples et faisant de la "maison pay- sanne" et du costume les emblèmes des cultures nationales régionales et locales. Ensuite l'impact de l'idéologie communiste dans la prise en compte de la "maison paysanne" a été déterminant dans cette idée de "construction nationale" (chapitre 1).

L'auteur cite Le Corbusier (chapitre 5) qui a une vision des origines de l'architecture où tout naturel est simple : "le plan de la maison naturelle est universel, ce ne sont que les moyens techniques qui évoluent." Cela traduit une pensée à la fois universaliste, ethnocentrique et évolutionniste, mais simpliste, dont témoignent également les textes de la charte d'Athènes [...]." Soit une vision occidentale moderne. Avec Le Corbusier on a affaire à un phénomène plus général de l'ordre du syndrome de la table rase, principe cartésien, fondateur de l'analyse moderne, c'est aussi un syndrome fréquent chez les révolutionnaires et les réformateurs. "La maison est un objet social qui fabrique du social et du sens ${ }^{13}$ dont la structure est en lien direct avec la culture, le lien entre structure et fonction sociale est déterminant jusqu'à Le Corbusier et l'invention de la "machine à habiter » type "HLM" (p.218-219). Généralement les théories évolutionnistes simplistes comme l'évolution linéaire idéale de l'habitation monocellulaire à la maison pluricellulaire ou de la maison enterrée à la maison à étages ne tiennent pas face à des études fines qui montrent que la complexité n'est pas absente des périodes anciennes. Un fonctionnalisme primaire à l'approche purement technologique et descriptive dont l'avantage est la simplicité et l'organicité, simplificateur et généralisant, a l'inconvénient de la tendance à la tautologie, la pauvreté interprétative culturelle et historique créant la confusion entre évolution et progrès (p.128).

Ce regard politique écrase les différences de statut social en milieu rural et oppose culture savante et culture populaire, les populations ne sont pas homogènes. C'est l'usage, la destination qui sont déterminants pour les choix formels. Pour être intéressante, la distinction entre populaire et savant risque fort de laisser entendre que ces deux modes 
de construire n'entretiennent aucun rapport. Or les emprunts réciproques sont constants.

La plupart des études classiques de l'architecture rurale mettent en avant sa diversité, son originalité. Dans le chapitre 3, F. Ruegg salue les travaux du Centre for Vernacular Architecture Studies d'Oxford pour leur approche comparatiste à grande échelle. Il dénonce l'approche des Hausforscher, aussi comparatistes, qui peuvent avoir eu l'intention de démontrer la supériorité ou la "véritable origine" d'un type de bâtiment «ethnique» ou national. Les chercheurs "grand-allemands » (Grossdeutsch) dans les années 1930 prenaient pour aire de recherche la partie orientale de l'Europe où des populations allemandes se sont installées au cours de l'histoire et tentèrent de démontrer la supériorité de l'architecture allemande du fait qu'elle avait été imitée ou importée par les populations locales, slaves notamment (p.113). Cette démarche peu glorieuse est scientifiquement contestable et on sait quelle idéologie les guidait.

Derrière les approches, technologiquefonctionnaliste ou diffusionniste, significatives des vicissitudes idéologiques de la «maisonologie» allemande depuis le début du $\mathrm{XX}^{\mathrm{e}}$ siècle se profile en fait le plus souvent une théorie évolutionniste primaire, attribuant le prétendu progrès de l'habitat rural soit à un peuple supérieur, soit au développement naturel des besoins au fil du temps.

Pour avoir lui-même pâti de ces lacunes théoriques, François Ruegg en appelle à une approche qui prenne en compte l'ensemble des conditions sociales et historiques. La notion de l'architecture populaire est en soi une invention. Le paysan qui aurait inventé sa propre architecture est un archétype du peuple allemand dans une vision ethniciste $^{14}$. Or les modèles autrichiens et allemands sont diffusés par la conquête à partir de la seconde moitié du XVIII ${ }^{e}$ siècle. Que certaines techniques comme par exemple la manière d'attacher les gerbes de chaume aux voliges, encore appelée par les paysans roumains « à l'allemande » aient certainement été introduites par les colons ne signifie en aucune manière qu'il y ait quelque supériorité que ce soit.

L'anthropologie culturelle apporte un éclairage par l'interprétation symbolique de l'espace domestique qui complète la compréhension de la «maison paysanne ».

La diversité et l'évolution des techniques ne peuvent pas rendre compte à elles seules de la forme de la construction et de la composition interne de l'habitat. Les descriptions et classifications de l'habitat selon les matériaux, les techniques, les formes, sans prise en compte des contextes socio-culturels et historiques de l'avènement de ces constructions reflètent plus le besoin du chercheur d'exorciser la diversité en reconstituant une hypothétique genèse, à moins qu'elle ne cède à la tentation folklorisante et ethnicisante de créer des types nationaux. La connaissance de techniques n'implique pas nécessairement leur emploi et le matériau ne dicte par nécessairement la forme.

Le déterminisme environnemental est une vieille explication de la différence culturelle, Rapoport s'en libère en parlant de "facteurs déterminants » (environnementaux) et de " facteurs modifiants » (techniques) dont les trois principaux sont repris par Ruegg dans le chapitre 4 :

- Le statut social du paysan et de son village (p.155-160) : organisation, économie, type de production agricole; histoire et démographie, morphologie des villages selon leur environnement topographique et l'aménagement du territoire ;

- La structure familiale et le système de parenté (p.16o-163): l'auteur prend notamment l'exemple de la Zadruga, structure communautaire traditionnelle typique de l'aire culturelle slave du sud, fonctionnant sur la base d'un regroupement familial à transmission patriarcale. Cette structure a été respectée, au moins en Serbie et constitue une forme marquante de l'habitat dispersé qui a naturellement représenté " l'origine naturelle et nationale du régime collectiviste » pour les tenants de l'idéologie communiste (p.160) ; 
- Le régime politique régissant les formes du territoire et modifiant les formes de l'architecture rurale (p.163-167): dans les Balkans, les divers courants architecturaux sont dus à la rencontre de populations d'origines différentes correspondant à des unités politiques et culturelles diverses : féodalisme, domination turque, domination austrohongroise puis collectivisme (chapitre 5). La colonisation s'est presque toujours accompagnée d'un programme de mise en valeur des terres, d'une organisation territoriale mettant en place le tissu d'exploitations rurales et des villages correspondant à l'idéologie entreprenariale de l'Aufklaerung, soit la création ou la "restructuration" (ou systématisation comme on aurait dit au temps de Ceausescu p.185) des villes et des villages. C'est l'Idealdorf. La colonisation autrichienne à partir de 1760 importe des idéaux urbanistiques dans l'organisation de l'exploitation des terroirs et l'organisation des ressources. Les modèles autrichien et allemand fondés sur la politique de conquête et d'organisation des territoires d'Europe centrale-orientale ont pour objectif de " civiliser le pays et ses habitants... ». La conquête, l'occupation et l'organisation du territoire sont liées à une culture politique. «Or la reconquête autrichienne des territoires occupés jusqu'au $17 \mathrm{e}$ siècle par les Ottomans permet d'observer la naissance, ou mieux la conception de la future "maison paysanne" célébrée ensuite comme le fleuron du génie national. » (p.178). Des manuels de construction rurale se multiplient dès le XVIII siècle $^{15}$, fonctionnalisme pratique de l'architecture seigneuriale à la campagne, ils suivent strictement les modèles de l'architecture savante. L'urbanisation éclairée des autorités impériales normalise la construction, l'Impopulation Hauptinstruktion de 1772 dicte de manière très précise la façon de construire une maison paysanne pour les colons ; ces normes servent de base à toute nouvelle construction rurale, coloniale ou non (p.186-193). Avec cette rationalisation des constructions rurales, se met en place un système de planification : coupes de bois, défrichement et préparation des terrains. Ainsi, l'habitat reflète une politique d'aménagement planifié. Les modèles n'existent pas seulement dans les formes mais correspondent également à une idéologie de la construction, conçue sur une base scientifique et politique, sujette à un enseignement académique et à une diffusion administrative. L'habitat correspond à des pratiques économiques et culturelles (élevage et type d'élevage; production agricole ; mobilité etc.) qui peuvent, elles aussi, être imposées par l'Etat. L'architecture vernaculaire est influencée par la puissance politique de manière plus ou moins forte, selon qu'il s'agit d'un pouvoir direct comme dans le cas des Habsbourg ou d'un pouvoir indirect (indirect rule) comme dans le cas des Ottomans.

Le chapitre 6 éclaire la contribution de l'anthropologie sociale et historique et permet de critiquer l'approche déterministe répandue chez les géographes. Alors que les Eskimos représentent une population «limite » défiant le climat, leur habitat n'est pas seulement lié aux conditions climatiques, il varie selon les saisons de même que les formes de la vie sociale ${ }^{16}$, des formes d'habitat avec variations saisonnières sont aussi connues en Europe centraleorientale jusqu'au XIX ${ }^{\mathrm{e}}$ siècle. Au-delà des divergences d'écoles, la perspective déterministe des géographes est limitée, bien que depuis la critique de M. Mauss, ces derniers ont changé de méthode et ne se cantonnent plus dans des explications telluriques.

L'histoire (histoire de l'art, mais surtout histoire sociale) politique et économique marquent l'habitat. L'organisation familiale et villageoise, l'appartenance à un Empire, le statut du paysan (libre ou servile), son statut économique, interviennent dans ces formes. En Europe occidentale, les structures sociales se sont ouvertes à partir du XVII ${ }^{e}$ siècle et surtout à partir de la Révolution française. Il faut toutefois faire la différence entre histoire de l'art et formes vernaculaires de l'art populaire. Il y a des interactions et des influences réciproques, l'architecture "savante" s'insère régulièrement dans l'architecture vernaculaire, particulièrement lorsque l'autorité est le fait d'un pouvoir politique fort. C'est 
la culture, génératrice de code social, qui engendre la forme.

Le développement de l'idée de nation est parallèle à la naissance de l'intérêt pour les identités rurales, s'ensuivent deux mouvements dans l'approche du vernaculaire: la patrimonialisation ou sauvegarde et la célébration des «cultures paysannes ». Dans les deux cas, il y a utilisation et rationalisation des techniques dites traditionnelles. « Le paysan éduqué et parfois prospère devient peu à peu digne d'intérêt car il incarne peu à peu la nation, "l'âme du peuple" » mais, comme le "primitif", il n'a pas voix au chapitre, tantôt considéré comme un "pauvre" sauvage à civiliser, tantôt comme un "bon sauvage à imiter". C'est de la confrontation entre ces deux approches que naît ce que Ruegg appelle le paradoxe de la double conversion, tendance qui continue au $\mathrm{XX}^{\mathrm{e}}$ siècle.

La maison est un système de symboles comprenant espaces sacrés et espaces symboliques, une image du monde dans une perception non linéaire du temps. L'idée d'architecture populaire nationale est parallèle à l'ethnocentrisme. Les symboles spatiaux et temporels dans l'habitat sont évoqués par des éléments appropriés, ainsi L'anthropomorphisme de la maison ou la maison-corps ${ }^{17}$. Une ethnologie engagée dans la sauvegarde des cultures rend compte des symboles, rites etc ; l'aspect communautaire des maisons longues du Néolithique remet en question l'idée de cellule originaire (noyau familial) des sociétés dites primitives. La transformation des formes de l'habitat est liée aux représentations collectives de la société ${ }^{18}$, cette idée permet de sortir d'un schéma strictement évolutionniste et linéaire de la société.

Les entreprises de classification et d'interprétation sont susceptibles de manipulations, ce qui les rend quelquefois douteuses et dangereuses. La "maison paysanne" peut être considérée soit comme « avantageusement traditionnelle et cultivée pour le cachet qu'elle peut prêter à un bâtiment... » soit comme archaïque, mais " c'est tout le dilemme de la conservation et de la mise en valeur du patrimoine
[...] » qui « devient l'enjeu non seulement de marchés immobiliers et patrimoniaux, mais également de représentations du monde qui correspondraient à des idiosyncrasies culturelles et techniques que l'on prend soin de répartir géographiquement en unités ethno-culturelles, renforcées par une communauté linguistique. »

Cet habitat demeure mal connu dans ses formes anciennes, à défaut d'analyses archéologiques poussées. L'histoire des techniques pourrait être un des éléments déterminants de la construction de typologies de l'habitat ; mais la "maison paysanne" participe d'une anthropologie de l'espace autant que de l'anthropologie de la maison telle que la souhaitait Rapoport. Cependant, préciser dans quelle mesure l'histoire des typologies architecturales et des usages de l'habitat pourrait relever de l'histoire des techniques n'est pas l'objet de ce livre, d'ailleurs l'histoire des techniques s'est relativement peu intéressée aux typologies de l'habitat rural (les classifications par type de toiture en France sont issues du travail des géographes) ${ }^{19}$. Ce livre se situe à la frontière des sciences humaines : ethnologie, histoire, géographie et archéologie. En cela, il adopte une approche anthropologique globale fondée sur un fait historique. Il met en avant l'importance dès la fin du XVIII ${ }^{\text {e }}$ siècle des écoles architecturales autrichiennes pour la constitution des canons architecturaux des maisons rurales d'Europe centrale orientale comme un véritable phénomène d'acculturation politique, en quelque sorte fondateur de l'idée de « maison paysanne » qui apparaît comme un objet ethnographique éclairé par l'anthropologie sociale ; un objet archéologique éclairé par l'histoire des techniques ; un objet historique éclairé par l'évolution des structures politiques ; un objet idéologique éclairé par les discours qui l'ont construit.

1 Il comporte également cinq encadrés, un cahier d'illustrations photographiques, et 33 figures dans le texte. En fin de volume, une dizaine de pages de bibliographie (soit 198 titres) ne repren- 
nent pas systématiquement les références citées dans le texte. Dans le cadre de ce commentaire on se permettra de rajouter quelques références illustrant spécifiquement nos propos ; on les distingue d'un *.

2 Pierre Clastres, Chroniques de indiens Guayaki, Paris, Plon, Terre humaine, $1972^{*}$ et La société contre l'Etat, Paris, Les éditions de minuit, $1974^{*}$.

3 Eugène Viollet-le-Duc Histoire de l'habitation humaine, Paris, Hetzel et Cie 1873- 1875 ; réimpression Pierre Madraga éditeur, Bruexelles, 1986.

4 Oeconomische Encyclopädie, oder allgemeines System der Staats- Stadt- Haus- u. Landwirtschaft de D. Johann Georg Krünitz, 1781 - livre 22, pp.258-308

5 Albert Deman-geon - "L'habitation rurale en France, essai de classification des principaux types ", Annales de géographie, 1920, vol.29, p.352 ; Albert Dauzat. : "Les anciens types d'habitation rurale en France. Leur répartition. Leur formation historique", La Nature, I, Paris, 1924, p.53-6o ; puis Corpus de l'architecture rurale française, de Demangeon à Cuisenier (1920 1980) Jean Cuisenier dir.*

6 Amos Rapoport, Hausform and culture, Englewoods Cliffs, New Jersey Prentice Hall, 1969 ; édition française : Pour une anthropologie de la maison, Paris, Dunod 1972.

7 André Leroi-Gourhan: Milieu et technique, Albin Michel, Paris, 1945-1973, $469 \mathrm{p}$.

8 Patricia Gaillard-Bans, Aspects de l'architecture rurale en Europe occidentale, Etablissements humains et socioculturel environnement $\mathrm{n}^{\circ} 15$, UNESCO 1978, $67 \mathrm{p}$.

9 p. 185 ; l'auteur utilise le terme d'Aufklaerung pour désigner la conquête germanique de l'Europe centrale-orientale engagée dans la seconde moitié du XVIII ${ }^{\mathrm{e}}$ siècle, analogie volontaire avec l'équivalent germanique du siècle des lumières et synthétisant clairement l'intention politique du mythe de la «maison paysanne ».

10 François Ruegg : A l'est rien de nouveau. De la Barbarie à la Civilisation ?, Genève, 1991.

11 "Bauernhaus das", in Oesterreich und seine Grenzgebieten, 1906, Dresden ; et "Bauernhaus das", in Deutschen Reiche und in seinen Randgebieten, 1906, Dresden

12 Vaclav Frolec, Lidova architektura na Moravea ve Slezsku. Brno, Blok, 1974, 399 p.,bibl., cartes, fig., ph.

13 p.146 ; citant A. Coudart : " Maisons Néolithiques, maisons de Nouvelle-Guinée. L'ethnologie comparée du choix social et technique », in De la Préhistoire aux missiles balistiques. L'intelli- gence sociale des techniques. B. Latour et P. Monnier ed. Paris, 1994, p.228-252.

14 Les travaux de la Revue de la Société d'Anthropologie de Vienne au début du XX $\mathrm{XX}^{\mathrm{e}}$ siècle (1902) comptent dans la mise en place de ces idées.

15 Cointereaux : Ecole d'architecture rurale ou leçons par lesquelles on apprendra soi-même à bâtir solidement les maisons à plusieurs étages avec la terre seule, Paris 1790

16 Référence à Marcel Mauss : « Essai sur les variations saisonnières des sociétés Eskimos, étude de morphologie sociale", L'année sociologique, T. IX 1904-1905; repris dans Sociologie et anthropologie, Paris PUF, 1968.

17 Bourdieu Pierre : "La maison kabyle», in Esquisse d'une théorie de la pratique précédé de trois études d'etnologie kabyle, Paris-Genève 1972.

18 A. Coudart, "Maisons néolithiques, maisons de NouvelleGuinée..." op. cit.

19 Par exemple, Lachiver Marcel - «Sur quelques aspects de la maison rurale en Seine-et-Oise au milieu du XIXe siècle. Nature des couvertures et hauteur des maisons ", Paris et Île-de-France, Mémoires publiés par la Fédération des Sociétés Historiques et Archéologiques de Paris et de l'île-de-France, 25, 1976, 73-85*, met en perspective une approche à la fois sociale et historique. 